\title{
Point-to-Set Distance Metric Learning on Deep Representations for Visual Tracking
}

\author{
Shengping Zhang, Yuankai Qi, Feng Jiang, Xiangyuan Lan, Pong C. Yuen, and Huiyu Zhou
}

\begin{abstract}
For autonomous driving application, a car shall be able to track objects in the scene in order to estimate where and how they will move such that the tracker embedded in the car can efficiently alert the car for effective collisionavoidance. Traditional discriminative object tracking methods usually train a binary classifier via a support vector machine (SVM) scheme to distinguish the target from its background. Despite demonstrated success, the performance of the SVM based trackers is limited because the classification is carried out only depending on support vectors (SVs) but the target's dynamic appearance may look similar to the training samples that have not been selected as SVs, especially when the training samples are not linearly classifiable. In such cases, the tracker may drift to the background and fail to track the target eventually. To address this problem, in this paper, we propose to integrate the point-toset/image-to-imageSet distance metric learning (DML) into visual tracking tasks and take full advantage of all the training samples when determining the best target candidate. The point-to-set DML is conducted on convolutional neural network features of the training data extracted from the starting frames. When a new frame comes, target candidates are first projected to the common subspace using the learned mapping functions, and then the candidate having the minimal distance to the target template sets is selected as the tracking result. Extensive experimental results show that even without model update the proposed method is able to achieve favourable performance on challenging image sequences compared to several state-of-the-art trackers.
\end{abstract}

Index Terms-metric learning, point to set, visual tracking.

\section{INTRODUCTION}

$\mathbf{V}$ ISUAL tracking has been attracting increasing interest in the past years [1]-[12] due to its important role in a wide range of applications such as video surveillance, visual reality, computer-human interaction, and autonomous driving, just to name a few. Among these applications, autonomous driving has various complications where moving targets are often mixed with significant background clutters. In addition, the measurement streams evolve in a complicated environment due to constantly changing appearance, severe occlusions and noise. Given a target in the first frame (usually in the format of a rectangle), its goal is to estimate the target states (e.g. position, scale, and rotation) throughout the remaining image frames. Despite lots of efforts have been devoted to

S. Zhang is with the School of Computer Science and Technology, Harbin Institute of Technology, Weihai 264209, China, e-mail: s.zhang@hit.edu.cn.

Y. Qi and F. Jiang are with the School of Computer Science and Technology, Harbin Institute of Technology, Harbin 150001, China, e-mail: \{yk.qi, fjiang\}@hit.edu.cn.

X. Lan and P. Yuen are with the Department of Computer Science, Hongkong Baptist University, Hong Kong, e-mail: \{lanxiangyuan, pcyuen\}@comp.hkbu.edu.hk.

H. Zhou is with the School of Electronics, Electrical Engineering and Computer Science, Queen's University of Belfast, Belfast, BT3 9DT, United Kingdom, E-mail: h.zhou@ecit.qub.ac.uk. visual tracking during the past decades, it still remains a challenging task due to distractions such as heavy occlusions, drastic illumination variations, large scale changes, out-ofplane rotations, fast motion, and severe camera shaking.

Broadly speaking, most of the existing tracking methods fall into either the generative or discriminative category. The generative methods cast object tracking as a searching task to find the best object candidate that is best represented by the target appearance model. Many methods have been proposed to robustly model the target appearance and account for illumination changes, occlusions, and view changes. Ross et al. [13] proposed to use the incremental subspace learning to adapt to target appearance changes. In [14], holistic sparse coding was introduced into visual tracking, where each positive dictionary item is a whole target template and trivial items are used to account for occlusions. Despite achieving good results, the holistic sparse model is not flexible enough to represent local variations of the target. To handle this problem, Jia et al. [15] proposed a local sparse coding based tracking method, where each dictionary item is a part region of the target template. As evidenced by their results, this scheme is able to encode the target appearance variations more flexibly and therefore obtains better performance. Later, a two-stage sparse coding strategy was proposed in [16] to combine the advantages of both the holistic and the local sparse coding models in order to obtain better performance. On the other hand, the discriminative methods formulate object tracking as a binary classification task, where the target region is viewed as the foreground and the remaining region is the background. These methods mainly focus on designing either discriminative features or powerful classifiers. For example, Danelljan et al. [17] proposed an adaptive low-dimensional feature based on color attributes, which is effective for color videos but not for black-white ones. In [9], the authors proposed an adaptive hedging method to combine different hierarchical features extracted from a deep convolutional neural network (CNN) and achieve reasonably better results than using only one type of feature. Different from these approaches, Hare et al. [18] proposed a novel classifier based on a kernelized structured output support vector machine (SVM), which directly embeds target positions into the objective function. Zhang et al. [19] designed a multi-expert tracking framework, which is able to filter out the appropriate expert with the most discriminative capability. More recently, several end-to-end CNN based tracking methods have been proposed, e.g. [10], [11], [20], to simultaneously learn discriminative features and effective classifiers.

Although recent discriminative tracking methods (e.g. [18], [19], [21]) usually obtain better results than generative ones, 


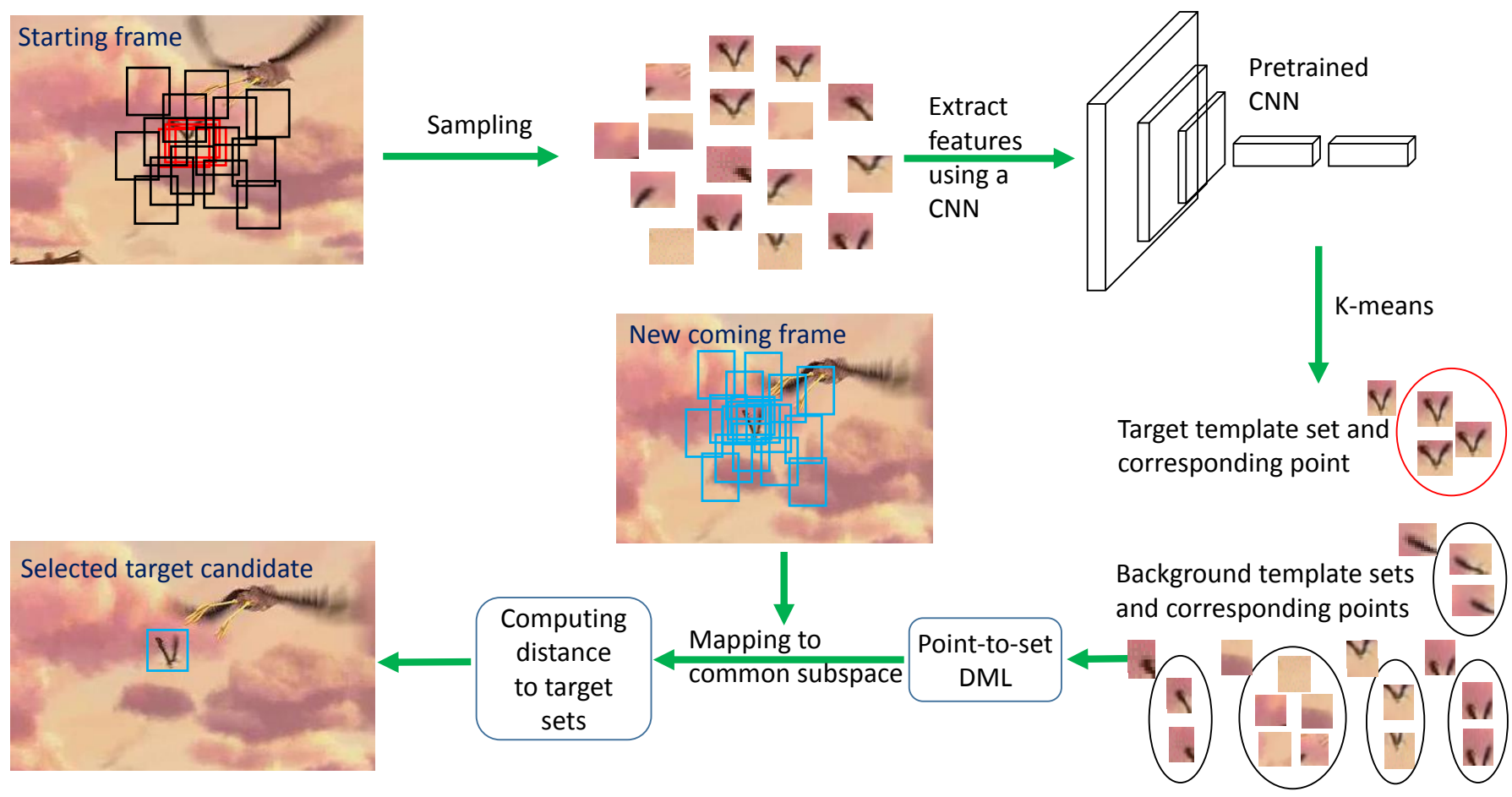

Fig. 1. Main steps of the proposed point-to-set distance metric learning based tracking algorithm. Target and background templates are randomly sampled from the starting frame. Then, we employ a pretrained CNN to compute their features. Based on the CNN features, these samples are divided into several target template sets and background template sets using a standard K-means method. Point-to-set distance metric learning (DML) is then performed on these sets (Section III-B). When a new frame comes, target candidates are randomly sampled and are projected to the common subspace using learned mapping functions. The candidate with the minimal distance to the target template sets is selected as the tracking result (Section III-D).

their performance is limited by the usage of SVMs. Because the SVMs determine the label of one test sample, only depending on the support vectors (SVs), the test sample may not always be correctly classified via SVs, especially when the training samples are not linearly classifiable. In such cases, the tracker may fail to find the best target candidate and therefore suffer from the tracking drift problem.

To address the above mentioned issue, we propose to take full advantages of all the training samples, instead of depending on only a few SVs, to measure the similarity between each target candidate and all the positive samples and the negative samples respectively via point-to-set distance metric learning. Specifically, we first cluster positive samples into several positive sets via K-means and cluster negative samples into negative sets in the same way to enhance the representative ability of each set. Then, for each training set we compute its mean feature vector of all the samples of that set, called mean sample. These mean samples represent the average appearances of the target and the background. We aim to learn mapping functions so as to pull the point-set pair with the same label close to each other and push far away otherwise. Since a mean sample can be viewed as a point in the high-dimensional Euclidean space and a sample set can be treated as a point on the Riemannian manifold, the above procedure can be formulated as mapping heterogeneous points on Euclidean space and Riemannian manifold into a common subspace. At the testing stage, all target candidates will firstly be projected onto the common subspace with the learned mapping functions and then the best target candidate is determined with a minimum distance to the positive sets. The main steps of the proposed point-to-set distance metric learning based tracking algorithm is illustrated in Figure 1.

The main contributions of this work are in two-fold:

1) We formulate the object tracking task as a point-to-set distance metric learning problem to take full advantages of all training samples.

2) Numerous experimental results on a large amount of challenging image sequences demonstrate the effectiveness of the proposed tracking method.

The remaining of this paper is organized as follows. In Section II, we present a discussion on the related work. In Section III, we give a detailed description of the proposed point-to-set metric learning based tracking algorithm. Then, we extensively evaluate the proposed tracker in Section IV. Finally, we conclude the work in Section V.

\section{RELATED WORK}

In this section, we briefly review some related visual tracking methods, which utilize convolutional neural networks (CNNs), distance metric learning (DML), sparse coding, and multiple cues for robust visual tracking.

With the great success of CNNs on tasks such as image classification [22]-[25], natural language processing [26], [27], object detection [28]-[30], and image segmentation [31], [32], several trackers based on CNNs have been proposed. Fan et al. [33] pre-train a network using the location and the appearance information of the object of interest to extract 
both spatial and temporal features. Hong et al. [34] learnt a discriminative appearance model using the $\mathrm{CNN}$ features of hidden layers and construct a target saliency map to help locate the target using the back-projecting CNN features. In [20], Wang et al. proposed a CNN architecture with two branches to simultaneously exploit high-level category information features captured by a top layer and low-level structure features captured by a lower layer. Nam and Han [10] designed a multidomain neural network to learn common representations using shared bottom layers and to learn discriminative representations using the top layer. Different from these CNN based trackers, which either use features extracted from only one layer or treat features extracted from different layers equally, Qi et al. [9] proposed an ensemble tracker that adaptively combines features extracted from as many as six different layers.

Many distance metric learning based trackers have been proposed to replace traditional hand-crafted metrics, such as the Euclidean distance, to better adapt to appearance variations. Wu et al. [35] learnt a distance metric over sparse representations of positive and negative training samples, which are further divided into several bags. However, they use the average distance of sample pairs from different bags as the distance between the corresponding bags, which incurs a high computational cost. Jiang et al. [2] used the sparsity regularization in metric learning to find the lowest possible dimensionality of the projection and thus to reduce the computational cost and also keep the best discriminative ability. In [36], Wang et al.unified the appearance modeling and visual matching using one objective in the formulation of metric learning to well capture the manifold structure of the discriminative appearances. In [1], Jiang et al. extended the linear metric to a nonlinear one using the RBF-kernel, and their training samples are collected on-the-fly, which makes the learned metric adaptive to appearance variations. Although the aforementioned DML based trackers improve the performance to some extent, they are still limited to the point-to-point/image-to-image metric learning. This not only incurs large computational cost but also may lead to visual mismatching when large appearance variation occurs. In this paper, we alleviate these problems by introducing the pointto-set/image-to-imageSet distance metric learning into visual tracking.

Besides the above mentioned approaches, there are also some methods that utilize multiple cues or learned representations to handle complex tracking scenarios. Optical flow, color, and depth clues are simultaneously incorporated in [37]. Edge information, intensity, and color features are combined into a discriminative one in [38]. Fang et al. [39] adds geometry constraint and attention selection to help associate tracklets. In [40], [41], both the RGB color and the depth information are combined to improve the performance of tracking. To obtain effective object representations, sparse coding and dictionary learning are used in [42]-[44]. He et al. [45] formulate association of two observations as a data association problem based on the spatial-temporal constraint that the trajectories of different objects must be disjoint.

\section{Proposed Algorithm}

In this section, we first give a brief description of some background of metric learning over Riemannian manifolds to put our method in a proper context. Then, we detail the proposed point-to-set distance metric learning based tracking algorithm.

\section{A. Background}

In the community of computer vision, a set of images can be treated as a point on the Symmetric Positive Definite (SPD) matrix manifold [46], Grassmannian manifold [47], or Affine Grassmannian manifold [48] depending on its representation in the usage of covariance matrices, linear subspaces, or affine subspaces, respectively. A prominent difference among these manifolds is the Riemannian metric over distance. In this paper, we base our algorithm on the SPD matrix manifold due to its better performance on point-to-set classification tasks than the other two as demonstrated in [49]. Let $S y m_{D}^{+}$denote the Riemannian manifold that has a Riemannian metric over the space of $(D \times D)$-dimensional symmetric positive definite matrices. According to the Log-Euclidean Distance [50], the distance between two points $P_{i}, P_{j} \in \mathbb{R}^{D \times D}$ on the manifold $\operatorname{Sym}_{D}^{+}$is defined as

$$
d\left(P_{i}, P_{j}\right)=\left\|\log \left(P_{i}\right)-\log \left(P_{j}\right)\right\|_{F},
$$

which is a true geodesic distance expressed by Euclidean computations. Classical Mahalanobis Distance is usually used to define the distance between an Euclidean point $x_{i}$ and a set $P_{j}$

$$
d\left(x_{i}, P_{j}\right)=\sqrt{\left(x_{i}-m_{j}\right)^{\top} P_{j}^{-1}\left(x_{i}-m_{j}\right)},
$$

where $m_{j}$ is the mean of all the samples in the dataset and $P_{j}$ resides on the $S y m_{D}^{+}$manifold.

\section{B. Learning Point-to-Set Distance Metric for Visual Tracking}

Let $X^{+}=\left\{x_{1}^{+}, \cdots, x_{m}^{+}\right\} \subset \mathbb{R}^{D}$ denote $m$ target templates in the form of vectorized features, and $X^{-}=$ $\left\{x_{1}^{-}, \cdots, x_{n}^{-}\right\} \subset \mathbb{R}^{D}$ denote $n$ background templates. These templates are extracted from the first frame of an image sequence at the beginning of tracking. A template having an overlap larger than 0.7 with the ground truth region is viewed as a target template. In contrast, a template having a overlap less than 0.5 is treated as a background template, which helps exclude incomplete target candidates.

Different from an SVM classifier, where only support vectors contribute to measuring the target candidates, in this paper, we introduce to learn a point-to-set distance metric [49] to take full advantage of all the training samples. To this end, we first cluster training templates into several sets, so that both the target and the background can be comprehensively described by representative appearances. Then, we learn the mapping functions that map Euclidean points and Riemannian manifold points into a common subspace. Because the gap between these two kinds of points are too large to bridge directly, we first project them to their own Hilbert spaces and then map to the common subspace, as shown in Figure 2. 


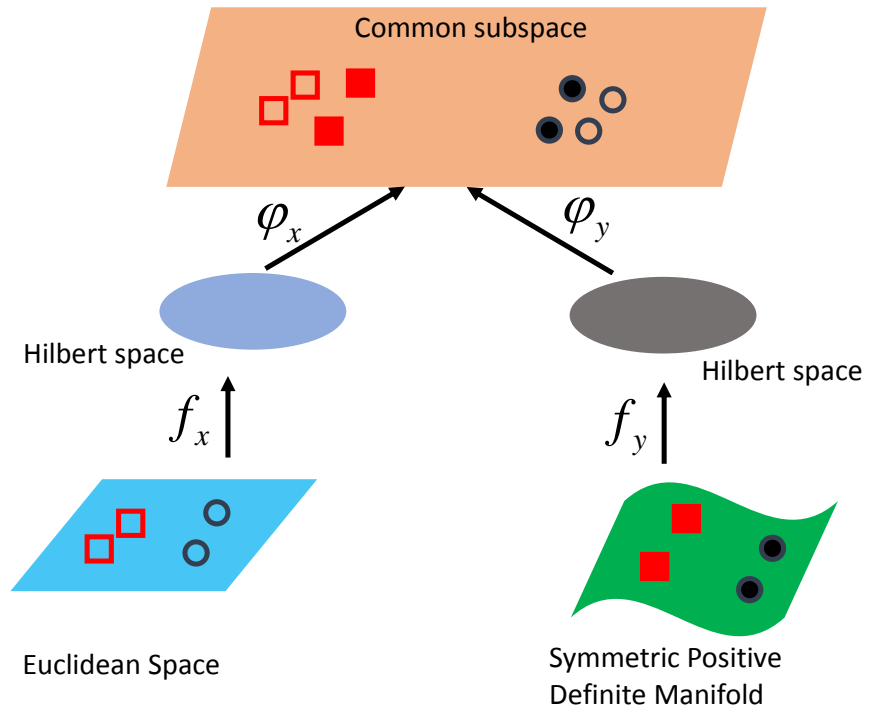

Fig. 2. Illustration of two-step point-to-set distance metric learning, where the original heterogeneous points are first mapped onto their own Hilbert space, and then map to the common subspace.

1) Constructing training data: We employ the traditional clustering method K-means to cluster the target templates in $X^{+}$into $m_{k}$ sets denoted by $S^{+}=\left\{S_{1}^{+}, \cdots, S_{m_{k}}^{+}\right\}$so as to minimize the within-cluster sum of squares of Euclidean distance of each point in the cluster to its cluster center

$$
\underset{S^{+}}{\arg \min } \sum_{i=1}^{m_{k}} \sum_{x \in S_{i}^{+}}\left\|x-\mu_{i}\right\|^{2},
$$

where $\mu_{i}$ is the mean of points in $S_{i}^{+}$. For each cluster, its mean vector is computed as its corresponding point in the Euclidean space, which is

$$
e_{i}^{+}=\frac{1}{\left|S_{i}^{+}\right|} \sum_{j \in S_{i}^{+}} x_{j}
$$

where $\left|S_{i}^{+}\right|$is the number of elements in set $S_{i}^{+}$. We totally obtain $m_{k}$ such Euclidean points denoted from $e_{1}^{+}$to $e_{m_{k}}^{+}$. The set $S_{i}^{+}$and its corresponding Euclidean point $e_{i}^{+}$are given the same label $l_{i}^{+}$. Similarly, we cluster the background templates $X^{-}$into $n_{k}$ sets denoted by $S^{-}=\left\{S_{1}^{-}, \cdots, S_{n_{k}}^{-}\right\}$and we compute their corresponding points in the Euclidean space denoted by $e_{1}^{-}, \cdots, e_{n_{k}}^{-}$. The label is accordingly denoted by $l_{1}^{-}, \cdots, l_{n_{k}}^{-}$. To embed the target sets in $S^{+}$and background sets in $S^{-}$into $S y m_{D}^{+}$manifold, each set is represented by its covariance matrix and is still denoted by the same symbol for simplicity from now on. Finally, we let $S=S^{+} \cup S^{-}$denote the union of $S^{+}$and $S^{-}$.

2) Objective formulation: With the above prepared training data, now we learn the mapping functions shown in Figure 2 to project these heterogeneous points into a common subspace by

$$
\underset{f, \varphi}{\arg \min } \lambda_{1} G_{e r}+\lambda_{2}\left(G_{e}+G_{r}\right)+\lambda_{3} R,
$$

where $G_{e r}$ is the constraint item defined on points projected from Euclidean space and Riemannian manifold $S y m_{D}^{+}, G_{e}$ is the constraint on points projected from Euclidean space, $G_{r}$ is the constraint on points projected from Riemannian manifold $\operatorname{Sym}_{D}^{+}$, and $R$ is the regularization item. The parameters $\lambda_{1}, \lambda_{2}$, and $\lambda_{3}$ control the trade-off among these three constraint items. Next, we detail these constraint items one by one.

Geometry constraint on points projected from heterogeneous spaces: $G_{e r}$ is defined to minimize the distance among points with the same label but projected from Euclidean space and Riemannian manifold $S y m_{D}^{+}$respectively:

$$
G_{e r}=\frac{1}{2} \sum_{i=1}^{m_{k}+n_{k}} \sum_{j=1}^{m_{k}+n_{k}} \mathbf{1}(i, j)\left\|f\left(x_{i}\right)-\varphi\left(S_{j}\right)\right\|^{2},
$$

where $x_{i} \in X^{+} \cup X^{-}, S_{j} \in S^{+} \cup S^{-}, \mathbf{1}(i, j)=1$ if $x_{i}$ and $S_{j}$ have the same label, and otherwise $\mathbf{1}(i, j)=-1$. This constraint ensures that points with the same label in heterogeneous space will be close to each other after the projection and the points will have a large distance if they are with different labels.

Geometry constraint on points projected from homogeneous spaces: The above item $G_{e r}$ only constrains the geometry relation of points mapped from heterogeneous space. However, the geometry relation of points from homogeneous spaces should also be taken into consideration. For that, we exert the following geometry constraints:

$$
\begin{aligned}
G_{e} & =\frac{1}{2} \sum_{i=1}^{m_{k}} \sum_{j=1}^{m_{k}} \mathbf{d}\left(x_{i}, x_{j}\right)\left\|f\left(x_{i}\right)-f\left(x_{j}\right)\right\|^{2}, \\
G_{r} & =\frac{1}{2} \sum_{i=1}^{n_{k}} \sum_{j=1}^{n_{k}} \mathbf{d}\left(S_{i}, S_{j}\right)\left\|f\left(S_{i}\right)-f\left(S_{j}\right)\right\|^{2},
\end{aligned}
$$

where $\mathbf{d}\left(v_{i}, v_{j}\right)=\exp \left(\left\|v_{i}-v_{j}\right\|^{2} / \sigma^{2}\right)$ if $v_{i}$ and $v_{j}$ are with the same label and they are $k_{1}$ neighborhood of one of them, $v$ denotes $x$ or $S$. If $v_{i}$ and $v_{j}$ have different labels but are $k_{2}$ neighborhood of one of them, $\mathbf{d}\left(v_{i}, v_{j}\right)=-\exp \left(\| v_{i}-\right.$ $\left.v_{j} \|^{2} / \sigma^{2}\right)$. For other cases, we define $\mathbf{d}\left(v_{i}, v_{j}\right)=0$.

Regularizer item $R$ : After projecting these heterogeneous points into a common subspace, the Euclidean distance will be employed as the measure metric. Thus, it is reasonable to equally treat all dimensions of these projected points. To this end, we impose the following constraint:

$$
R=\frac{1}{2}\left(\sum_{i=1}^{m_{k}+n_{k}}\left\|f\left(x_{i}\right)\right\|^{2}+\sum_{j=1}^{m_{k}+n_{k}}\left\|\varphi\left(S_{j}\right)\right\|^{2}\right) .
$$

\section{Optimization}

Although finding the global optimal solution for the objective function Eq. 5 is intractable, we can seek an approximate optima using the alternative iterative optimization method as shown in [49], which is capable of yielding desirable results as demonstrated in experiments. Let $\varphi_{x}\left(x_{i}\right)=V_{x}^{\top} x_{i}$, $\varphi_{s}\left(S_{i}\right)=V_{s}^{\top} S_{i}$. Then, by using the kernel trick, we get $\varphi_{x}\left(f_{x}\left(x_{i}\right)\right)=V_{x}^{\top} f_{x}\left(x_{i}\right)=\sum_{j} W_{x}^{\top} f_{x}\left(x_{j}\right) f_{x}\left(x_{i}\right)=$ $W_{x}^{\top} K_{x}\left(\cdot, x_{i}\right)$, where $K_{x}\left(x_{i}, x_{j}\right)=\left\langle f_{x}\left(x_{i}\right), f_{x}\left(x_{j}\right)\right\rangle$. Similarly, we can get $\varphi_{s}\left(f_{s}\left(S_{i}\right)\right)=W_{s}^{\top} K_{s}(\cdot, i)$, where $K_{s}\left(S_{i}, S_{j}\right)=\left\langle f_{s}\left(S_{i}\right), f_{s}\left(S_{j}\right)\right\rangle$. With these notations, the 
constraint items in Eq. 5 can be re-formulated in the matrix format:

$$
\begin{aligned}
G_{e r}\left(W_{x}, W_{s}\right)= & \frac{1}{2}\left(W_{x}^{\top} K_{x} \hat{B}_{x} K_{x}^{\top} W_{x}+W_{s}^{\top} K_{s} \hat{B}_{s} K_{s}^{\top} W_{s}\right. \\
& \left.-2 W_{x}^{\top} K_{x} Z K_{s} W_{s}^{\top}\right), \\
G_{e}\left(W_{x}\right)= & W_{x}^{\top} K_{x} B_{x} K_{x}^{\top} W_{x}-W_{x}^{\top} K_{x} Z_{x} K_{x}^{\top} W_{x} \\
= & W_{x}^{\top} K_{x} L_{x} K_{x}^{\top} W_{x}, \\
G_{r}\left(W_{s}\right)= & W_{s}^{\top} K_{s} B_{s} K_{s}^{\top} W_{s}-W_{s}^{\top} K_{s} Z_{s} K_{s}^{\top} W_{s} \\
= & W_{s}^{\top} K_{s} L_{s} K_{s}^{\top} W_{s},
\end{aligned}
$$

where $\hat{B}_{x}, \hat{B}_{s}, B_{x}$ and $B_{s}$ are diagonal matrices with

$$
\begin{gathered}
\hat{B}_{x}(i, i)=\sum_{j=1}^{n_{k}} \mathbf{1}(i, j), \quad \hat{B}_{s}(i, i)=\sum_{j=1}^{m_{k}} \mathbf{1}(j, i), \\
B_{x}(i, i)=\sum_{j=1}^{m_{k}} \mathbf{d}\left(x_{i}, x_{j}\right), \quad B_{S}(i, i)=\sum_{j=1}^{n_{k}} \mathbf{d}\left(S_{i}, S_{j}\right) .
\end{gathered}
$$

Let $v$ denotes $x$ or $S$, if $l_{i}^{v}=l_{j}^{v}$ and $v_{i}$ and $v_{j}$ are $k_{1}$ neighborhood, $Z_{v}(i, j)=\mathbf{d}(i, j)$; if $l_{i}^{v} \neq l_{j}^{v}$ and $v_{i}$ and $v_{j}$ are $k_{2}$ neighborhood, $Z_{v}(i, j)=-\mathbf{d}(i, j)$; otherwise, $Z_{v}(i, j)=0$. Then, the object function Eq. 5 can be re-written as function of $W_{x}$ and $W_{s}$ :

$$
\underset{W_{x}, W_{s}}{\arg \min } \lambda_{1} G_{e r}\left(W_{x}, W_{s}\right)+\lambda_{2}\left(G_{e} W_{x}+G_{r} W_{s}\right)+\lambda_{3} R .
$$

Given an initialization of $W_{s}$, the $W_{x}$ can be updated by differentiating Eq. 14 with respect to $W_{x}$ and setting it to zeros. Here we directly give the analytical solution as follows:

$$
W_{x}=\left(K_{x}\left(\hat{B}_{x}+2 \lambda_{1} L_{x}+2 \lambda_{2} I\right) K_{x}^{\top}\right)^{-1} K_{x} Q K_{s}^{\top} W_{s}
$$

where $Q(i, j)=\mathbf{1}(i, j)$. With the updated $W_{x}$ in hand, a new $W_{s}$ is able to obtained in a similar way, namely setting the partial differential of Eq. 14 with respect to $W_{s}$ to zeros. The $W_{s}$ is updated as

$$
W_{s}=\left(K_{s}\left(\hat{B}_{s}+2 \lambda_{1} L_{s}+2 \lambda_{2} I\right) K_{s}^{\top}\right)^{-1} K_{s} Q K_{x}^{\top} W_{x} .
$$

\section{Tracking with the learned distance metric}

In the above section, we have learned the mapping functions using the samples extracted from the first frame of a test image sequence to project points from Euclidean space and Riemannian manifold $S y m_{D}^{+}$into a common subspace. Based on these mapping functions, when a new frame comes, we perform the following steps to determine the new position and scale of the target:

Step 1: In the $t$-th frame sampling target candidates $y_{1}, \cdots, y_{q}$ under the Gaussian distribution $\mathbf{G}_{p}\left(\mu_{p}, \sigma_{p}\right)$, where

$$
\begin{aligned}
& \mu_{p}=\left(p_{x}^{t-1}, p_{y}^{t-1}\right), \\
& \sigma_{p}=1,
\end{aligned}
$$

and $\left(p_{x}^{t-1}, p_{y}^{t-1}\right)$ is the target position in the $(t-1)$-th frame. The sampling scales $N_{s}$ subject to

$$
N_{s}=N_{o} \times 1.05^{\max (-1, \min (1,5 \times \operatorname{Gaussian}(0,1)))},
$$

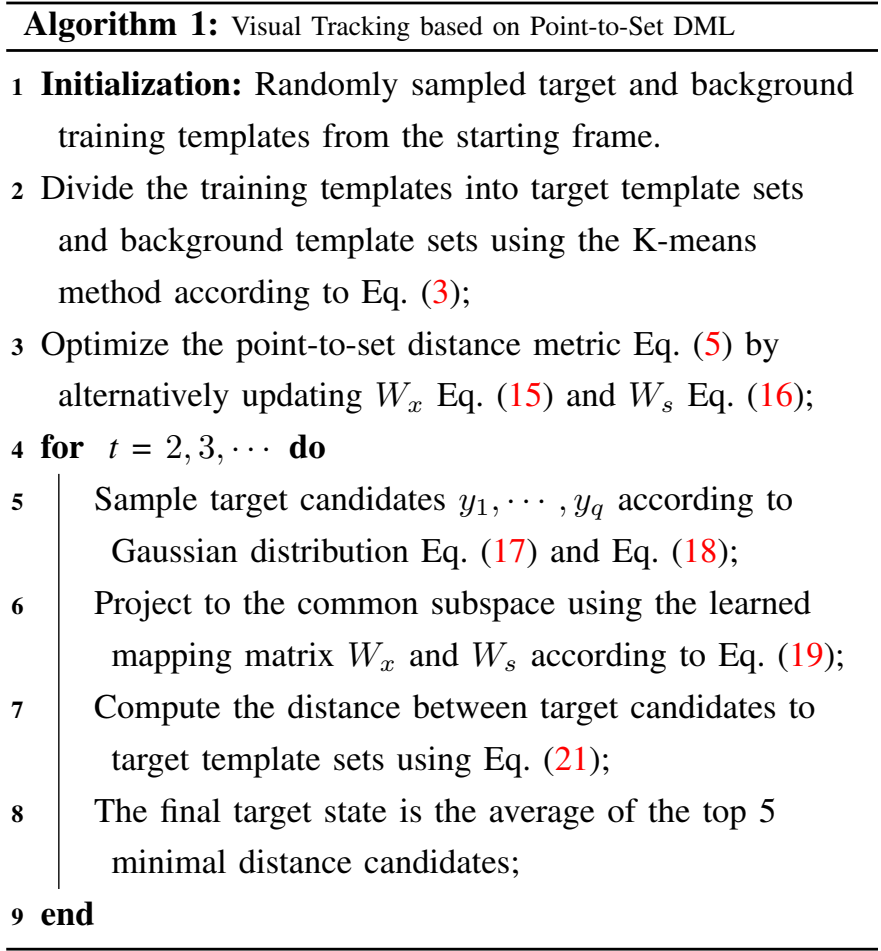

where $N_{o}$ denotes the scale of the target in the last frame. Then, features of these candidates are computed as doing in the first frame. For simplicity, these features are still denoted by $Y=y_{1}, \cdots, y_{q}$.

Step 2: Mapping target candidates, target template sets, and background sets to the common subspace,

$$
\begin{aligned}
\hat{Y} & =W_{x}^{\top} K_{y}=W_{x}^{\top}\left[\hat{K}_{y}, \hat{K}_{y, s}\right], \\
\hat{S} & =W_{s}^{\top} K_{s}=W_{s}^{\top}\left[\hat{K}_{s}, \hat{K}_{y, s}^{\top}\right],
\end{aligned}
$$

where

$$
\begin{aligned}
\hat{K}_{y}(i, j) & =\exp \left(-\left\|y_{i}-y_{j}\right\|^{2} /\left(2 \sigma^{2}\right)\right) \\
\hat{K}_{s}(i, j) & =\exp \left(-\left\|\log \left(S_{i}\right)-\log \left(S_{j}\right)\right\|^{2} /\left(2 \sigma^{2}\right)\right) \\
\hat{K}_{y, s}(i, j) & =\exp \left(-\left(y_{i}-e_{j}\right)^{\top} S_{j}^{-1}\left(y_{i}-e_{j}\right) /\left(2 \sigma^{2}\right)\right) .
\end{aligned}
$$

Step 3: In the common subspace computing the distance between target candidates and target template sets $\hat{S}_{1}^{+}, \cdots, \hat{S}_{m_{k}}^{+}$ according to Eq. 21, where $\hat{S}_{i}^{+}$is the counterpart of $S_{i}^{+}$in $\hat{S}$.

$$
d\left(y_{i}, S^{+}\right)=\sum_{j=1}^{m_{k}} d\left(y_{i}, S_{j}^{+}\right)=\sum_{j=1}^{m_{k}}\left(1-\frac{\hat{Y}_{i}^{\top} \hat{S}_{j}^{+}}{\left\|\hat{Y}_{i}^{\top}\right\|_{2}\left\|\hat{S}_{j}^{+}\right\|_{2}}\right) .
$$

Step 4: The final target state is the average of the top 5 minimal distance candidates. We summarize the main steps of the proposed tracking algorithm in Alg. 1.

\section{EXPERIMENTS}

In this section, we first describe the implementation details and then quantitatively and qualitatively evaluate the proposed Point-to-Set distance metric learning based Tracker (PST) on 31 publicly used challenging sequences. These videos are full of challenges such as heavy illumination variations, partial or 
TABLE I

COMPARISON OVER THE AVERAGE OVERLAP RATE (THE LARGER THE BETTER). THE BEST AND THE SECOND BEST RESULTS ARE SHOWN IN RED AND BLUE COLORS, RESPECTIVELY.

\begin{tabular}{|c|c|c|c|c|c|c|c|c|c|c|c|c|c|c|c|c|c|c|}
\hline & PST & Struck & SCM & IVT & L1APG & MTT & CXT & Frag & TLD & DLT & ASLA & DFT & LOT & LSST & MEEM & DSST & DAT & PPT \\
\hline Boy & 821 & 760 & 377 & 0.260 & 0.734 & 0.500 & 0.542 & 0.389 & 0.662 & .828 & 0.369 & 0.401 & 0.533 & 0.358 & 0.797 & 0.138 & 0.680 & 0.805 \\
\hline Suv & 63 & 19 & & 406 & 476 & 449 & 736 & 628 & 0.693 & 787 & 0.495 & 0.079 & 0.654 & .611 & 0.627 & 0.148 & .098 & 0.540 \\
\hline Tiger1 & 597 & 153 & 157 & 0.095 & 0.314 & 0.260 & 0.322 & .265 & 0.376 & 0.372 & 0.290 & 0.532 & 0.139 & 0.094 & 0.649 & 0.213 & 0.415 & 0.586 \\
\hline Tiger2 & 525 & 0.544 & 0.090 & 0.086 & 0.244 & 0.287 & 0.359 & 0.118 & 0.262 & 0.214 & 0.145 & 0.681 & 0.135 & 0.088 & 0.540 & 0.149 & 0.448 & 0.556 \\
\hline Walking & 604 & 0.572 & 0.711 & 0.766 & 0.753 & 0.666 & 0.171 & 0.537 & 0.446 & 0.413 & 0.772 & 0.562 & 0.704 & 0.757 & 0.540 & 0.159 & 0.547 & 0.701 \\
\hline CarDark & 595 & 0.892 & 0.843 & 0.663 & 0.884 & 0.827 & 0.566 & 0.301 & 0.449 & 0.588 & 0.849 & 0.380 & 0.420 & 0.835 & 0.862 & 0.177 & 0.031 & 0.533 \\
\hline Deer & 725 & 740 & 071 & 0.033 & 602 & 0.612 & 0.697 & .173 & 0.602 & .041 & 0.033 & 0.256 & 0.198 & 0.033 & 0.745 & 0.148 & 0.118 & 0.709 \\
\hline Dudek & 346 & 730 & 769 & 0.753 & 692 & 0.758 & 0.731 & 0.535 & 0.648 & 0.791 & 0.737 & 0.690 & 0.536 & 0.796 & 0.715 & 0.142 & 0.170 & 0.851 \\
\hline FaceOcc2 & 553 & 0.785 & 0.728 & 0.727 & 0.687 & 0.745 & 0.742 & 0.648 & 0.616 & 0.654 & 0.646 & 0.768 & 0.458 & 0.463 & & 0.130 & 0.273 & 0.659 \\
\hline Football1 & 592 & 0.669 & 0.396 & 0.557 & 0.557 & 0.556 & 0.763 & 0.364 & 0.377 & 0.343 & 0.493 & 0.867 & 0.536 & 0.480 & 0.680 & 0.352 & 0.661 & 0.686 \\
\hline Girl & 716 & 0.746 & 0.682 & 0.167 & 0.730 & 0.665 & 0.554 & 0.454 & 0.572 & .543 & 0.711 & 0.283 & 0.418 & 0.151 & 0.698 & 0.249 & 0.460 & 0.704 \\
\hline Joggi & 621 & 0.171 & 178 & 0.176 & 74 & 0.177 & 0.766 & 0.518 & 0.770 & 181 & 0.179 & 0.187 & 0.092 & 0.175 & 0.670 & 0.036 & 0.177 & 0.754 \\
\hline & 596 & 617 & 122 & 0.122 & 150 & 0.097 & 0.524 & .672 & 0.664 & 580 & 0.227 & 0.110 & 0.578 & 0.126 & 0.708 & 0.010 & 0.048 & 0.649 \\
\hline & 561 & 412 & 319 & 0.226 & 99 & 0.207 & 0.257 & 0.330 & 0.518 & .348 & 0.247 & 0.222 & 0.828 & 0.207 & 0.789 & 0.161 & 0.335 & 0.555 \\
\hline Matrix & 452 & 0.098 & 0.255 & 0.024 & 0.147 & 0.233 & 0.058 & 0.057 & 0.156 & 0.012 & 0.121 & 0.058 & 0.111 & 0.083 & & & 0.068 & 0.306 \\
\hline Mhyang & 757 & 0.818 & 0.809 & 0.796 & 0.829 & 0.853 & 0.850 & 0.651 & 0.632 & 0.887 & 0.916 & 0.709 & 0.215 & 0.834 & 0.790 & 0.167 & 0.591 & 0.810 \\
\hline MotorRo & 461 & 0.154 & 0.111 & 0.089 & 0.093 & 0.090 & 0.129 & 0.118 & 0.229 & 0.091 & 0.106 & 0.084 & 0.121 & 0.098 & 0.110 & 0.029 & 0.091 & 0.576 \\
\hline & 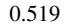 & & 368 & 74 & & 0.340 & & 05 & 0.725 & & & & 0.194 & 0.877 & & & 0.335 & 0.788 \\
\hline Blur & 754 & 846 & 411 & 0.145 & 67 & 0.780 & 0.764 & 0.560 & 0.717 & 0.685 & 0.380 & 0.717 & 0.561 & 0.528 & 0.795 & 0.153 & 0.807 & 0.398 \\
\hline Humar & 584 & 007 & 006 & 0.007 & & 0.006 & 0.011 & 0.015 & 0.006 & .007 & 0.007 & 0.007 & 0.011 & 0.007 & 0.527 & 0.004 & 0.065 & 0.045 \\
\hline Board & 749 & 0.652 & 0.575 & 0.191 & 0.132 & 0.198 & 0.291 & 0.512 & 0.091 & 0.538 & 0.309 & 0.325 & 0.209 & 0.236 & 0.612 & 0.122 & 0.044 & 0.752 \\
\hline Car24 & 487 & 0.139 & 0.862 & 0.663 & 0.776 & 0.854 & 0.787 & 0.115 & 0.568 & 0.769 & 0.783 & 0.077 & 0.290 & 0.857 & 0.433 & 0.236 & 0.357 & 0.643 \\
\hline Dog & 0.521 & 0.344 & 0.391 & 0.131 & & 0.383 & 0.645 & 0.334 & 0.590 & 0.471 & 0.518 & 0.311 & 0.453 & 0.097 & 0.355 & 0.108 & 0.356 & 0.494 \\
\hline KiteS & 0567 & 640 & 267 & 0.287 & & 0.298 & & 0.126 & 0.337 & & & & 0.029 & & & & 0.242 & 0.698 \\
\hline Man & 775 & 98 & 328 & 0.750 & 852 & 0.882 & 0.8 & 1 & 0.739 & 6 & 0.850 & 0.202 & 0.185 & 0.803 & 0.831 & 0.176 & 0.506 & 0.802 \\
\hline & U & & 0.492 & 0.106 & & 0.163 & & 29 & 0.397 & 52 & 0.502 & 0.2 & 0.041 & 0.224 & & 0.067 & 0.556 & 0.555 \\
\hline RedTean & 631 & 0.497 & 0.482 & 0.629 & 0.570 & 0.538 & 0.380 & 0.138 & 0.354 & 0.698 & 0.617 & 0.272 & 0.100 & 0.407 & 0.511 & 0.162 & 0.459 & 0.635 \\
\hline Skater & 0.559 & 0.632 & 0.519 & 0.552 & 0.605 & 0.628 & 0.623 & 0.509 & 0.434 & 0.578 & 0.564 & 0.359 & 0.445 & 0.533 & 0.572 & 0.145 & 0.568 & 0.556 \\
\hline Skating2-2 & 0.464 & 0.385 & 0.400 & 0.094 & & 0.061 & 0.058 & 0.207 & 0.046 & & 0.282 & 0.052 & 0.366 & 0.076 & 0.378 & 0.046 & 0.137 & 0.489 \\
\hline & 0.707 & & 0.395 & 0.069 & & 0.101 & & & 0.674 & & & & & & & & & 0.745 \\
\hline Twinnings & 0.705 & 0.579 & 0.520 & 0.414 & 0.582 & 0.441 & 0.436 & 0.471 & 0.352 & 0.657 & 0.555 & 0.485 & 0.504 & 0.691 & 0.567 & 0.139 & 0.487 & 0.583 \\
\hline Average & 0.622 & 0.524 & 0.464 & 0.341 & 0.449 & 0.440 & 0.495 & 0.345 & 0.474 & 0.482 & 0.456 & 0.347 & 0.335 & 0.386 & 0.602 & 0.131 & 0.341 & 0.618 \\
\hline
\end{tabular}

full occlusions, abrupt camera shaking, large scale changes, in plane or out-of-plane rotation, large object deformation, and heavy motion blur, to name a few.

The proposed trackers are compared to 16 state-of-theart trackers: correlation filter based tracker with scale estimation (DSST) [51], distractor-aware tracker (DAT) [52], multi-expert based tracker (MEEM) [19], structured output tracking (Struck) [18], holistic and local sparsity based tracker (SCM) [16], tracking-learning-detection tracker (TLD) [53], adaptive local sparse appearance model based tracker (ASLA) [54], multi-task sparse learning based tracker (MTT) [55], deep learning based tracker (DLT) [56], context tracker (CXT) [57], fragments based tracker (Frag) [58], incremental learning based tracker (IVT) [59], $\ell_{1}$ sparse tracker using APG (L1APG) [60], distribution fields for tracking (DFT) [61], locally orderless tracking (LOT) [62], and least soft-threshold squares tracking (LSST) [63]. In addition, we make a comparison to an ITML [64] based tracker, denoted as PPT. ITML is employed to learn a distance metric in the case, which uses the cluster center to replace the image set in each training pair.

The average success rate and average center location error are employed to measure the performance in the quantitative evaluations following the recently developed algorithms [16], [54], [56], [65]. The average success rate is defined as $\frac{1}{N} \sum_{i=1}^{N} \frac{B_{i}^{t} \cap B_{i}^{g t}}{B_{t}^{t} \cup B_{i}^{g t}}$, where $B_{i}^{t}$ is the tracked result at the $i$ th frame by the tracker in the format of a rectangle bounding box, $B_{i}^{g t}$ is the ground truth at the $i$ th frame, $N$ is the total number of frames in the test image sequence. The average center location error is the average Euclidean distance in pixels between center points of the tracked bounding box and the ground truth.

\section{A. Implementation details}

To start the proposed tracker, $m=500$ positive examples and $n=5000$ negative examples are sampled in the first frame to learn the mapping functions. These examples are all resized to $107 \times 107$ pixels. Each example is represented by a 512-dimensional vector extracted by the output of the FC6 layer of the convolutional neural network MDNet [10]. Then, we cluster these examples into $m_{k}=7$ target template sets and $n_{k}=20$ background template sets using the standard Kmeans method. The values of $m_{k}$ and $n_{k}$ are determined to seek a balance between the computation cost and the tracking accuracy. The trade-off parameters $\lambda_{1}, \lambda_{2}$, and $\lambda_{3}$ are set to $1,0.01$, and 0.1 , respectively, which are determined by grid search in a wide range as shown in Table II. The neighborhood number $k_{1}$ and $k_{2}$ are both set to 1 . When optimizing the objective, we find that the alternative iteration number 30 can lead to a desirable solution.

\section{B. Quantitative evaluation}

Table I shows the performance comparison on 31 challenging image sequences in terms of the average overlap rate. From Table I we can observer that the proposed PST ranks top two on most of the sequences, which demonstrates its favourable performance compared to other state-of-the-art trackers. In particular, our tracker achieves an improvement of more than 20 percent over the second best one on the MotorRolling sequences, where the tracking target undergoes the combination of heavily rotations, illumination changes, 

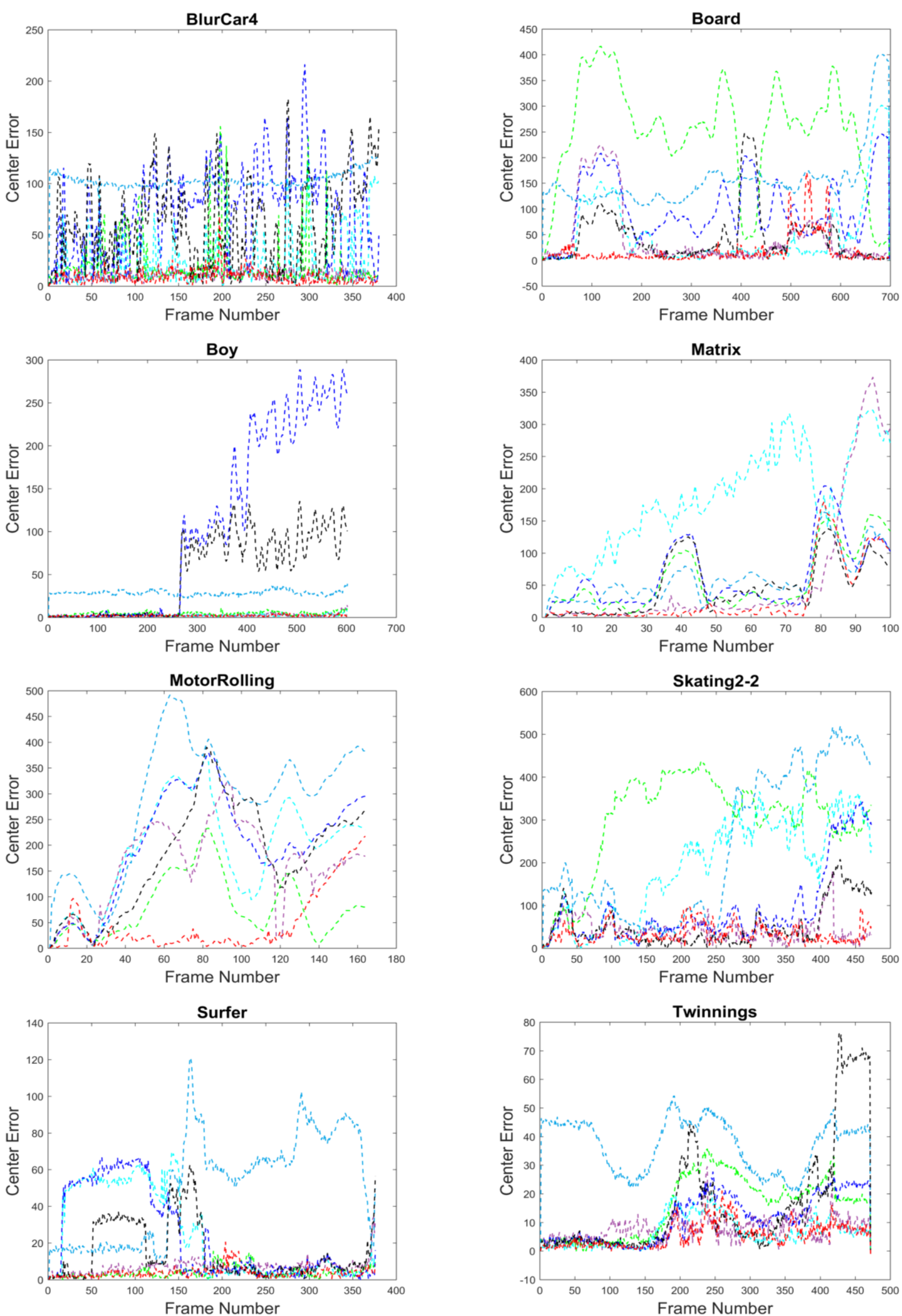

--- DLT --- MEEM --- DSST --- TLD --- ASLA --- SCM --- PST

Fig. 3. Frame-by-frame center location errors of our tracker PST against other six state-of-the-art trackers on several challenging image sequences. 

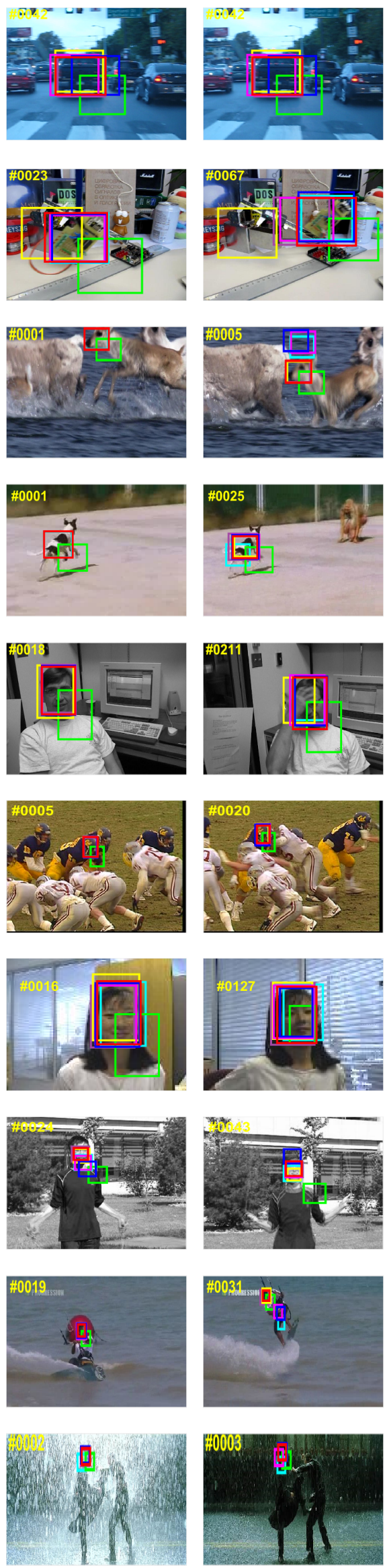

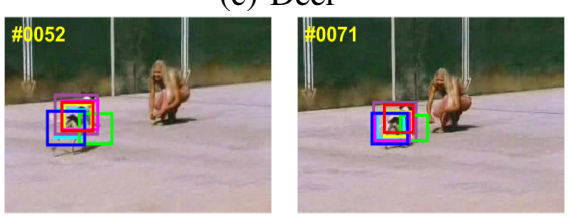

(c) Deer

(a) BlurCar4

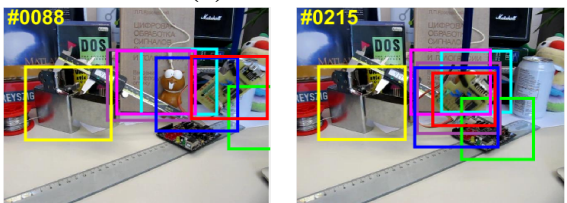

(b) Board

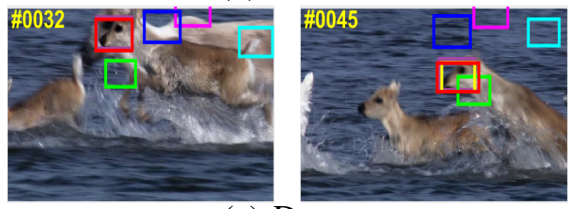

(d) Dog
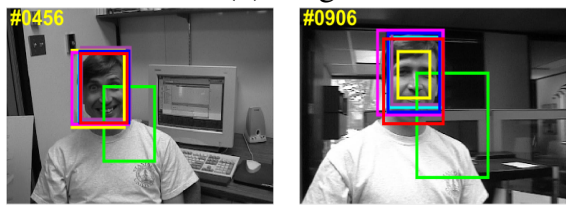

(e) Dudek
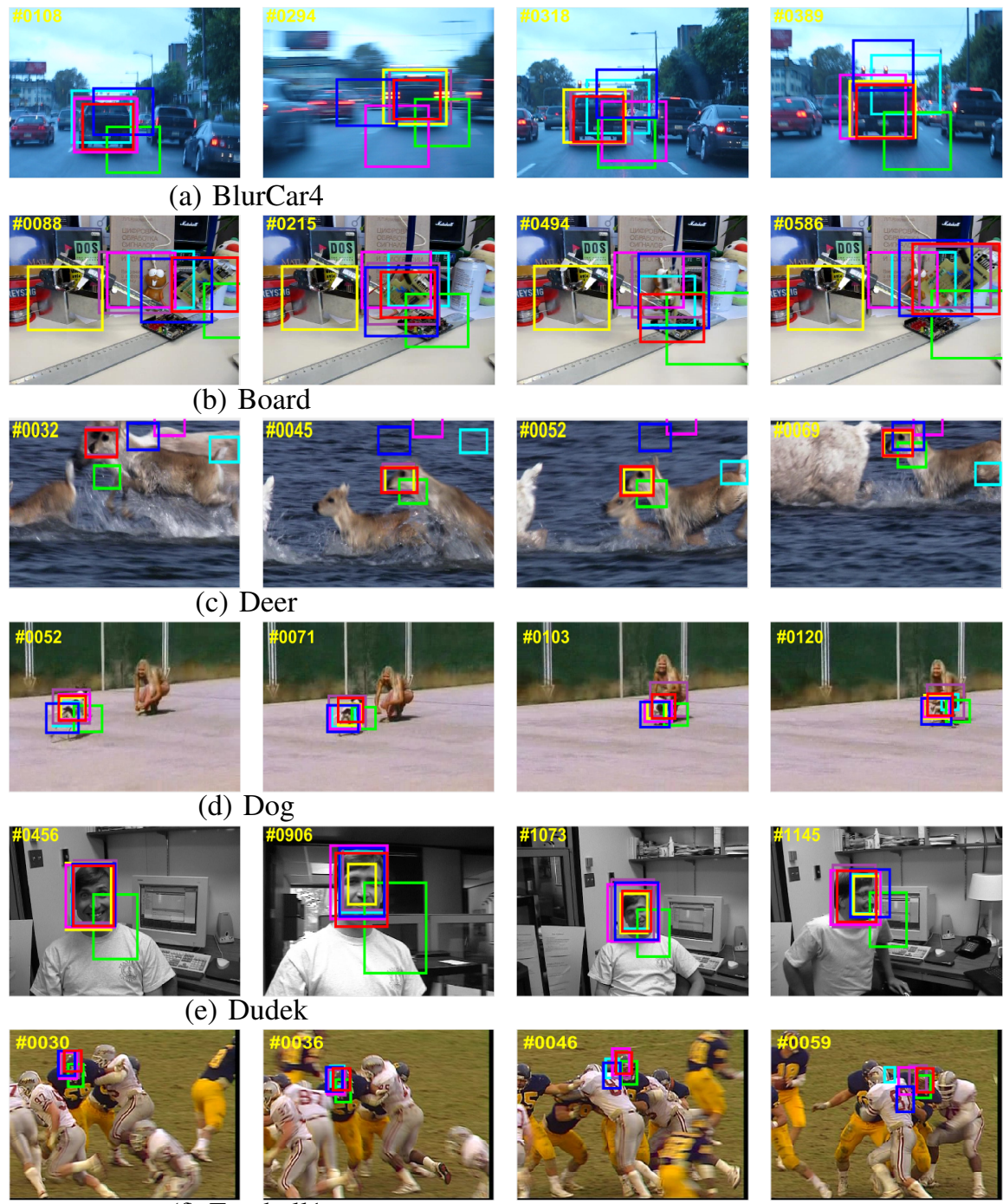

(f) Football1
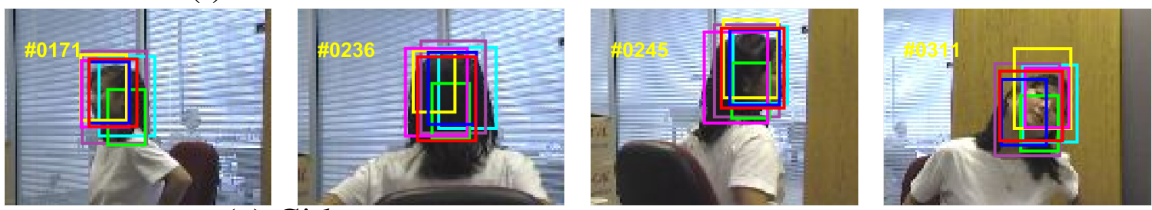

(g) Girl
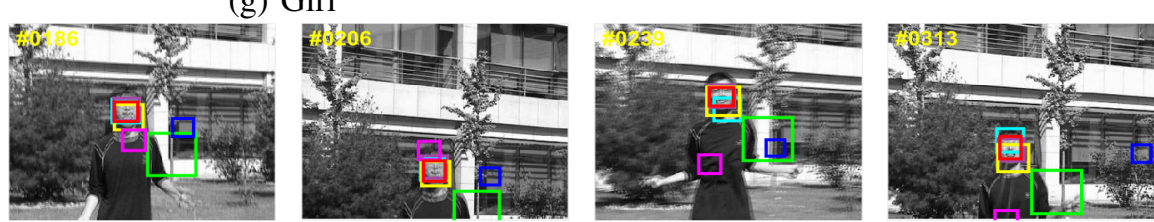

(h) Jumping
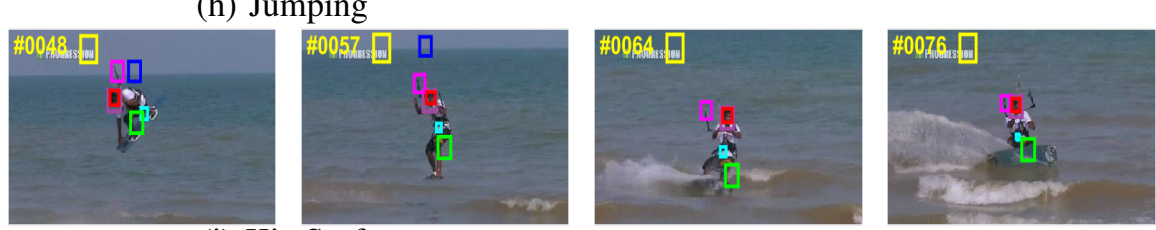

(i) KiteSurf
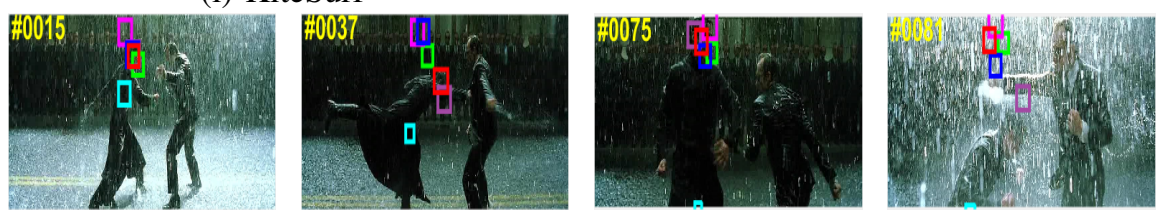

(j) Matrix 
TABLE II

TRACKING PERFORMANCE IN TERMS OF AVERAGE BOUNDING BOX OVERLAP OBTAINED BY VARYING THE KEY PARAMETERS $\lambda_{1}, \lambda_{2}$, AND $\lambda_{3}$ IN A LARGE RANGE.

\begin{tabular}{c|ccc|ccc|ccc}
\hline & \multicolumn{3}{|c|}{$\lambda_{1}=0.01$} & \multicolumn{3}{c|}{$\lambda_{1}=0.1$} & \multicolumn{3}{c}{$\lambda_{1}=1$} \\
\hline & $\lambda_{3}=0.01$ & $\lambda_{3}=0.1$ & $\lambda_{3}=1$ & $\lambda_{3}=0.01$ & $\lambda_{3}=0.1$ & $\lambda_{3}=1$ & $\lambda_{3}=0.01$ & $\lambda_{3}=0.1$ & $\lambda_{3}=1$ \\
\hline$\lambda_{2}=1$ & 0.416 & 0.269 & 0.221 & 0.375 & 0.275 & 0.059 & 0.404 & 0.311 & 0.123 \\
$\lambda_{2}=0.1$ & 0.247 & 0.116 & 0.419 & 0.305 & 0.151 & 0.422 & 0.361 & 0.142 & 0.470 \\
$\lambda_{2}=0.01$ & 0.186 & 0.492 & 0.364 & 0.149 & 0.457 & 0.435 & 0.144 & 0.622 & 0.493 \\
\hline
\end{tabular}

TABLE III

COMPARISON OVER THE AVERAGE CENTER LOCATION ERROR IN PIXELS (THE SMALLER THE BETTER). THE BEST AND THE SECOND BEST RESULTS ARE SHOWN IN RED AND BLUE COLORS, RESPECTIVELY.

\begin{tabular}{|c|c|c|c|c|c|c|c|c|c|c|c|c|c|c|c|c|c|c|}
\hline & PST & Struck & SCM & IVT & L1APG & MTT & CXT & Frag & TLD & DLT & ASLA & DFT & LOT & LSST & MEEM & DSST & DAT & PPT \\
\hline Boy & 1.9 & 3.8 & 51.0 & 91.3 & 7.0 & 12.8 & 7.4 & 40.5 & 4.5 & 2.3 & 106.1 & 106.3 & 66.0 & 109.3 & 2.5 & 28.4 & 6.1 & 2.1 \\
\hline Suv & 63.7 & 49.8 & 4.6 & 57.3 & 93.1 & 180.7 & 9.9 & 42.0 & 13.0 & 4.4 & 74.8 & 111.4 & 29.5 & 33.4 & 29.8 & 51.8 & 110.1 & 48.4 \\
\hline Tiger1 & 19.2 & 128.7 & 93.5 & 106.6 & 58.4 & 64.4 & 45.4 & 74.3 & 49.5 & 37.2 & 55.9 & 41.3 & 111.4 & 111.8 & 13.7 & 48.8 & 31.6 & 19.7 \\
\hline Tiger2 & 24.6 & 21.6 & 141.2 & 105.1 & 65.2 & 48.8 & 41.4 & 113.5 & 37.1 & 48.5 & 85.8 & 12.2 & 150.4 & 81.1 & 19.5 & 61.5 & 30.0 & 20.0 \\
\hline Walking & 4.0 & 4.6 & 2.5 & 1.6 & 3.3 & 3.5 & 205.7 & 9.3 & 10.2 & 15.4 & 1.9 & 5.9 & 2.4 & 1.6 & 4.8 & 30.3 & 6.9 & 3.4 \\
\hline CarDark & 7.9 & 1.0 & 1.2 & 8.4 & 1.0 & 1.6 & 16.5 & 36.5 & 27.5 & 18.8 & 1.5 & 58.8 & 30.9 & 1.4 & 1.3 & 17.2 & 104.7 & 10.6 \\
\hline Deer & 6.2 & 5.3 & 103.5 & 182.7 & 24.2 & 18.9 & 6.7 & 105.1 & 30.9 & 236.7 & 160.1 & 98.7 & 65.2 & 232.7 & 4.7 & 53.8 & 143.9 & 5.9 \\
\hline Dudek & 8.6 & 11.4 & 10.8 & 9.6 & 23.5 & 14.1 & 12.8 & 82.7 & 18.1 & 8.8 & 15.3 & 18.7 & 85.1 & 9.1 & 14.6 & 117.0 & 199.5 & 8.0 \\
\hline FaceOcc2 & 12.1 & 6.0 & 9.0 & 7.4 & 12.8 & 9.8 & 6.3 & 16.0 & 12.3 & 10.9 & 19.3 & 7.9 & 14.9 & 10.6 & 6.8 & 60.4 & 46.7 & 18.0 \\
\hline Football1 & 9.8 & 5.4 & 20.3 & 24.5 & 9.2 & 12.7 & 2.6 & 15.7 & 45.4 & 25.7 & 12.2 & 2.0 & 6.8 & 11.5 & 5.1 & 15.9 & 5.4 & 8.8 \\
\hline Girl & 3.3 & 2.6 & 2.5 & 22.5 & 2.8 & 4.3 & 11.0 & 20.7 & 9.8 & 10.1 & 3.3 & 24.0 & 22.8 & 26.7 & 3.6 & 16.2 & 15.2 & 6.6 \\
\hline Jogging-1 & 15.6 & 62.1 & 132.8 & 86.1 & 89.5 & 108.0 & 5.6 & 21.5 & 6.7 & 108.0 & 104.6 & 31.4 & 90.9 & 95.5 & 6.2 & 107.3 & 92.8 & 7.5 \\
\hline Jumping & 5.3 & 6.5 & 65.9 & 61.6 & 83.7 & 84.6 & 10.0 & 5.6 & 5.9 & 8.7 & 46.1 & 67.1 & 5.6 & 60.4 & 4.4 & 78.3 & 50.7 & 5.6 \\
\hline Liquor & 59.4 & 91.0 & 99.2 & 118.5 & 212.9 & 543.4 & 131.8 & 99.6 & 37.6 & 113.2 & 146.7 & 221.1 & 8.5 & 166.7 & 11.3 & 126.4 & 171.9 & 58.7 \\
\hline Matrix & 31.7 & 194.5 & 48.2 & 124.4 & 61.7 & 75.4 & 151.6 & 181.5 & 57.2 & 184.0 & 65.2 & 105.8 & 73.5 & 111.4 & 60.5 & 61.2 & 107.5 & 49.2 \\
\hline Mhyang & 1.9 & 2.6 & 2.4 & 1.9 & 3.2 & 3.1 & 4.0 & 12.5 & 9.5 & 2.4 & 1.7 & 9.1 & 113.1 & 2.1 & 4.5 & 44.8 & 14.8 & 1.6 \\
\hline MotorRolling & 45.6 & 145.7 & 177.6 & 174.7 & 207.0 & 191.1 & 131.8 & 146.2 & 80.9 & 193.0 & 201.4 & 174.2 & 135.4 & 144.9 & 163.7 & 286.5 & 145.1 & 17.2 \\
\hline Singer1 & 26.8 & 14.5 & 2.7 & 11.3 & 53.4 & 36.2 & 11.4 & 88.9 & 8.0 & 3.0 & 3.3 & 18.8 & 141.4 & 2.8 & 29.7 & 85.1 & 35.5 & 3.4 \\
\hline BlurCar4 & 8.9 & 4.9 & 63.6 & 133.7 & 6.6 & 13.6 & 8.8 & 56.8 & 20.1 & 27.2 & 71.5 & 18.5 & 26.9 & 44.5 & 10.8 & 102.9 & 10.5 & 118.9 \\
\hline Human3 & 16.6 & 230.6 & 521.1 & 227.9 & 283.9 & 428.6 & 164.9 & 224.2 & 249.4 & 232.4 & 249.4 & 227.1 & 226.6 & 245.4 & 13.6 & 348.3 & 188.8 & 196.1 \\
\hline Board & 17.9 & 33.6 & 44.5 & 150.2 & 173.2 & 147.6 & 126.0 & 91.4 & 248.4 & 52.1 & 100.9 & 99.3 & 182.0 & 84.7 & 44.4 & 156.0 & 319.8 & 18.0 \\
\hline Car24 & 73.0 & 119.7 & 1.9 & 1.4 & 2.1 & 2.6 & 3.5 & 127.1 & 3.0 & 1.9 & 3.0 & 165.6 & 100.0 & 2.2 & 4.9 & 17.6 & 12.1 & 12.0 \\
\hline Dog & 6.0 & 10.4 & 11.1 & 93.4 & 9.2 & 6.1 & 4.6 & 12.2 & 6.1 & 9.3 & 5.3 & 15.8 & 10.3 & 38.0 & 7.2 & 28.6 & 7.3 & 5.8 \\
\hline KiteSurf & 4.8 & 6.1 & 66.2 & 53.8 & 43.5 & 54.7 & 36.8 & 141.1 & 58.6 & 37.3 & 25.9 & 32.4 & 84.4 & 45.5 & 3.3 & 45.8 & 59.0 & 3.3 \\
\hline Man & 2.7 & 1.4 & 1.9 & 2.9 & 1.3 & 1.5 & 2.2 & 57.0 & 3.4 & 1.4 & 1.5 & 39.9 & 33.1 & 1.9 & 2.3 & 21.1 & 9.7 & 2.2 \\
\hline Panda & 5.2 & 7.2 & 7.2 & 79.8 & 50.9 & 20.2 & 64.0 & 61.7 & 32.1 & 7.0 & 7.4 & 48.5 & 78.3 & 31.7 & 7.4 & 43.6 & 6.0 & 5.1 \\
\hline RedTeam & 3.4 & 4.3 & 4.1 & 3.3 & 4.0 & 2.7 & 16.8 & 54.9 & 35.5 & 3.0 & 3.3 & 50.3 & 71.9 & 45.0 & 3.5 & 19.3 & 4.9 & 3.5 \\
\hline Skater & 10.3 & 8.2 & 13.2 & 8.7 & 14.5 & 7.8 & 7.6 & 24.3 & 12.8 & 10.2 & 7.8 & 46.0 & 9.5 & 11.7 & 11.8 & 81.0 & 16.6 & 9.7 \\
\hline Skating2-2 & 33.7 & 75.4 & 51.4 & 198.2 & 198.5 & 196.7 & 213.2 & 126.8 & 299.5 & 189.9 & 90.7 & 236.7 & 51.0 & 203.3 & 42.2 & 234.5 & 171.3 & 31.4 \\
\hline Surfer & 3.7 & 9.0 & 14.8 & 63.5 & 101.4 & 37.0 & 3.1 & 51.6 & 3.8 & 24.0 & 22.9 & 150.9 & 26.1 & 23.4 & 7.0 & 50.6 & 7.1 & 3.2 \\
\hline Twinnings & 5.5 & 5.5 & 16.9 & 13.8 & 8.1 & 15.0 & 21.3 & 16.9 & 15.6 & 6.8 & 11.5 & 12.4 & 20.5 & 5.7 & 7.9 & 37.2 & 16.5 & 16.0 \\
\hline Average & 17.4 & 41.1 & 57.6 & 71.8 & 61.6 & 75.7 & 47.9 & 69.6 & 46.9 & 52.7 & 55.0 & 72.8 & 66.9 & 64.4 & 17.8 & 79.9 & 69.3 & 23.2 \\
\hline
\end{tabular}

TABLE IV

COMPARISON OF RUNNING SPEED (THE LARGER THE BETTER). THE BEST AND THE SECOND BEST RESULTS ARE SHOWN IN RED AND BLUE COLORS, RESPECTIVELY

\begin{tabular}{|c|c|c|c|c|c|c|c|c|c|c|c|c|c|c|c|c|c|c|}
\hline & & & 4 & IVT & & UTT & $\mathrm{T}$ & Frag & CLD & OLT & $\mathrm{A}$ & $\mathrm{FT}$ & LT & $\mathrm{ST}$ & & ST & $\mathrm{AT}$ & PPT \\
\hline & 17 & 14 & 36 & 7.24 & .94 & 0.79 & 3.67 & 4.60 & 0.66 & 8.68 & 7.55 & 1.05 & 0.48 & .33 & 21.32 & 3.48 & 2.43 & 64 \\
\hline
\end{tabular}

and deformations. On the most challenging sequence Matrix, where the appearance of the tracking target is severely interfered by thick darkness, thunder, and heavy rain, the majority of the compared trackers achieves an average overlap rate lower than 0.1 . In contrast, our tracker attains up to 0.45 , which is 6 percent higher than the second best.

Table III presents the average center location error comparisons, where a smaller value means better performance. It can be seen that the proposed PST method is able to track targets with a relatively smaller spatial errors on most of the sequences. Taking the Board image sequence for example, the target undergoes complicated challenges including scale variations, out-of-view, motion blur, and out-of-plane rotation of $360^{\circ}$, which makes tracking the target a very tough task. Our tracker achieves the minimal average center location error with 17.9 pixels, which is 15.7-pixel smaller than the second best one. In such a challenging scenario, with the leading average overlap rate in Table I on the Matrix image sequence, our tracking result here is also 25.5-pixel more precise than the second best. Overall, from Table III and Table I we can see that our tracker performs favorably against other state-ofthe-art methods in terms of both criteria.

To make a more comprehensive evaluation of the proposed tracker, in Figure 3 we show the detailed frame-by-frame center location errors of our tracker compared to other state-ofthe-art trackers on several challenging image sequences. From these plots we can see that the proposed PST can achieve consistent lower center location errors than the other trackers, which demonstrates the robustness and the effectiveness of our tracker. The effectiveness of the proposed tracking algorithm can be attributed to two aspects: (1) Given a specific task, the specially learned distance metric for this task is usually better than the Euclidean distance. (2) The adopted deep convolutional features generally are more discriminative than 
traditional hand-crafted features.

Table IV presents the run-time of evaluated tracking methods. The results show that DAT and DSST run very fast with FPS larger than 50, and the proposed tracking method runs at 0.17 FPS. Taking also the tracking accuracy into consideration, although our PST runs slow, it achieves a more than $30 \%$ higher performance than the fastest trackers DAT and DSST in terms of the average overlap rate as shown by the last row of Table I and more than 50 pixels preciser in terms of the average center local error metric as shown by the last row of Table III. Compared to other trackers running at a similar speed such as SCM, MTT, and LOT, the proposed tracking method PST also has at least a $10 \%$ improvement as shown by Table I and III.

\section{Qualitative evaluation}

In addition to the quantitative evaluation, we also evaluate the proposed algorithm qualitatively against other state-of-theart trackers on several challenging sequences. We present the qualitative bounding box comparisons in Figure 4.

1) Out-of-plane Rotation: The target in the Girl image sequence poses a tough tracking task that contains a $360^{\circ}$ outof-plane rotation, which shows the front face, the side face, the back side of the head, and the other side face in turn. As shown in Figure $4(\mathrm{~g})$, our PST method is able to locate the target precisely even when the slide face and the back of the head appear as shown in frames 171, 236, and 245, although our training examples are sampled only from the first frame and keep unchanged throughout the whole sequences. This can be attributed to the learned point-to-set distance metric, which exploits all the training samples in the target template set and the background template set to measure the distance to a target candidate. While there are no new target templates added to the training sets, the existing background templates including blue windows, yellow wooden wall, and white Tshirt can be used to push candidates with background regions away by the learned point-to-set distance metric, which shows the advantage of exploiting the rich features of all the training samples rather than the fixed support vectors in SVMs. In contrast, other state-of-the-art trackers, such as MEEM, DSST, and ASLA, encounter drifts to some extent.

2) Occlusion, Scale Variation, and Deformation: As shown in Figure 4 (e), the target of the Dudek image sequence undergoes significant occlusions (more than half of the face is occluded by his hand, see frame 211), scale variation, and deformations (such as facial expression changes and with/without eyeglasses). We can observe that the proposed PST method achieves very good results in these cases, as shown in frames $211,906,1073$, and 1145 . This can be attributed to the learned point-to-set distance metric. Specifically, taking the occluded frame 211 for example, although the occluded face with a hand does not appears in the target template sets, it neither appears in the background templates set. Thus, candidates with occluded face are still able to obtain a relatively large distance to the background set compared to candidates that have an overlap with the background. As a result, the occluded target can be distinguished from backgrounds. Except the TLD tracker, the remaining trackers also perform very well in the presence of occlusions.

3) Severe Blur and Cluttered Background: As shown in frame 294 and frame 239 of Figure 4 (a) and (h) respectively, the targets in sequences BlurCar4 and Jumping are severely blurred in the cluttered background due to either abrupt fast motion of the camera or the fast motion of the target itself. From the results we can see that our tracker can handle these challenges well. In contrast, other state-of-the-art trackers, such as SCM and DSST, fail to locate the target after heavy blur occurs.

\section{CONCLUSION}

This paper presents a novel tracking algorithm based on the point-to-set distance metric learning for object tracking in autonomous driving applications. The point-to-set distance metric was learned by minimizing the intra-class distance and meanwhile maximizing the inter-class distance in the common subspace after a two-step projection. With the learned distance metric, we are able to measure each target candidate with the reference to all the training samples instead of some fixed support vectors in SVMs. Numerous experimental results on challenging image sequences demonstrate the effectiveness of the proposed method compared to other state-of-the-art trackers.

\section{ACKNOWLEDGMENT}

This work was supported in part by the National Natural Science Foundation of China (Nos. 61300111 and 61672188). H. Zhou is also supported by UK EPSRC under Grants EP/G034303/1, EP/N508664/1 and EP/N011074/1.

\section{REFERENCES}

[1] N. Jiang, W. Liu, and Y. Wu, "Learning adaptive metric for robust visual tracking," TIP, vol. 20, pp. 2288-2300, 2011.

[2] — "Order determination and sparsity-regularized metric learning adaptive visual tracking," in CVPR, 2012.

[3] S. Zhang, H. Yao, X. Sun, and S. Liu, "Robust visual tracking using an effective appearance model based on sparse coding," ACM Transactions on Intelligent Systems and Technology, vol. 3, no. 3, pp. 1-18, 2012.

[4] S. Zhang, H. Yao, H. Zhou, X. Sun, and S. Liu, "Robust visual tracking based on online learning sparse representation," Neurocomputing, vol. 100, no. 1, pp. 31-40, 2013.

[5] S. Zhang, H. Yao, X. Sun, and X. Lu, "Sparse coding based visual tracking: Review and experimental comparison," Pattern Recognition, vol. 46, no. 7, pp. 1772-1788, 2013.

[6] S. Zhang, H. Zhou, F. Jiang, and X. Li, "Robust visual tracking using structurally random projection and weighted least squares," IEEE Transactions on Circuits and Systems for Video Technology, vol. 25, no. 11, pp. 1749-1760, 2015.

[7] L. Zhang, W. Wu, T. Chen, N. Strobel, and D. Comaniciu, "Robust object tracking using semi-supervised appearance dictionary learning," Pattern Recognition Letters, vol. 62, pp. 17-23, 2015.

[8] S. Zhang, H. Zhou, H. Yao, Y. Zhang, K. Wang, and J. Zhang, "Adaptive normalhedge for robust visual tracking," Signal Processing, vol. 110, pp. 132-142, 2015.

[9] Y. Qi, S. Zhang, L. Qin, H. Yao, Q. Huang, J. Lim, and M. Yang, "Hedged deep tracking," in CVPR, 2016.

[10] H. Nam and B. Han, "Learning multi-domain convolutional neural networks for visual tracking," in CVPR, 2016.

[11] R. Tao, E. Gavves, and A. W. M. Smeulders, "Siamese instance search for tracking," in CVPR, 2016.

[12] X. Lan, S. Zhang, and P. C. Yuen, "Robust joint discriminative feature learning for visual tracking," in Proceedings of International Joint Conference on Artificial Intelligence, 2016, pp. 3403-3410. 
[13] D. A. Ross, J. Lim, R.-S. Lin, and M.-H. Yang, "Incremental learning for robust visual tracking," International Journal of Computer Vision, vol. 77, pp. 125-141, 2008.

[14] X. Mei and H. Ling, "Robust visual tracking using 11 minimization," in Proceedings of the IEEE International Conference on Computer Vision, 2009.

[15] X. Jia, H. Lu, and M.-H. Yang, "Visual tracking via adaptive structural local sparse appearance model," in CVPR, 2012.

[16] W. Zhong, H. Lu, and M.-H. Yang, "Robust object tracking via sparsitybased collaborative model," in CVPR, 2012.

[17] M. Danelljan, F. S. Khan, M. Felsberg, and J. van de Weijer, "Adaptive color attributes for real-time visual tracking," in CVPR, 2014.

[18] S. Hare, A. Saffari, and P. H. S. Torr, "Struck: Structured output tracking with kernels," in Proceedings of the IEEE International Conference on Computer Vision, 2011.

[19] J. Zhang, S. Ma, and S. Sclaroff, "MEEM: robust tracking via multiple experts using entropy minimization," in Proceedings of European Conference on Computer Vision, 2014.

[20] L. Wang, W. Ouyang, X. Wang, and H. Lu, "Visual tracking with fully convolutional networks," in ICCV, 2015.

[21] S. Hong, T. You, S. Kwak, and B. Han, "Online tracking by learning discriminative saliency map with convolutional neural network," in ICML, 2015

[22] C. Szegedy, W. Liu, Y. Jia, P. Sermanet, S. E. Reed, D. Anguelov, D. Erhan, V. Vanhoucke, and A. Rabinovich, "Going deeper with convolutions," in CVPR, 2015.

[23] K. He, X. Zhang, S. Ren, and J. Sun, "Deep residual learning for image recognition," in CVPR, 2016.

[24] K. Simonyan and A. Zisserman, "Very deep convolutional networks for large-scale image recognition," CoRR, vol. abs/1409.1556, 2014.

[25] L. Wang, B. Zhang, J. Han, L. Shen, and C. Qian, "Robust object representation by boosting-like deep learning architecture," Sig. Proc.: Image Comm., vol. 47, pp. 490-499, 2016.

[26] A. Kumar, O. Irsoy, P. Ondruska, M. Iyyer, J. Bradbury, I. Gulrajani, V. Zhong, R. Paulus, and R. Socher, "Ask me anything: Dynamic memory networks for natural language processing," in ICML, 2016.

[27] R. Collobert and J. Weston, "A unified architecture for natural language processing: deep neural networks with multitask learning," in $I C M L$, 2008.

[28] S. Ren, K. He, R. Girshick, and J. Sun, "Faster R-CNN: Towards realtime object detection with region proposal networks," in NIPS, 2015.

[29] J. Dai, Y. Li, K. He, and J. Sun, "R-FCN: object detection via regionbased fully convolutional networks," in NIPS, 2016.

[30] D. Zhang, J. Han, J. Han, and L. Shao, "Detection based on intrasaliency prior transfer and deep intersaliency mining," IEEE Trans. Neural Netw. Learning Syst., vol. 27, no. 6, pp. 1163-1176, 2016.

[31] J. Dai, K. He, and J. Sun, "Instance-aware semantic segmentation via multi-task network cascades," in CVPR, 2016.

[32] J. Long, E. Shelhamer, and T. Darrell, "Fully convolutional networks for semantic segmentation," in CVPR, 2015

[33] J. Fan, W. Xu, Y. Wu, and Y. Gong, "Human tracking using convolutional neural networks," TNN, vol. 21, 2010.

[34] S. Hong, T. You, S. Kwak, and B. Han, "Online tracking by learning discriminative saliency map with convolutional neural network," in $I C M L, 2015$

[35] Y. Wu, B. Ma, M. Yang, J. Zhang, and Y. Jia, "Metric learning based structural appearance model for robust visual tracking," TCSVT, vol. 24, pp. 865-877, 2014.

[36] X. Wang, G. Hua, and T. X. Han, "Discriminative tracking by metric learning," in $E C C V, 2010$

[37] Q. Wang, J. Fang, and Y. Yuan, "Multi-cue based tracking," Neurocomputing, vol. 131, pp. 227-236, 2014

[38] X. Li, Q. Liu, Z. He, H. Wang, C. Zhang, and W. Chen, "A multi-view model for visual tracking via correlation filters," Knowl.-Based Syst., pp. 88-99, 2016.

[39] J. Fang, Q. Wang, and Y. Yuan, "Part-based online tracking with geometry constraint and attention selection," TCSVT, vol. 24, pp. 854 864, 2014

[40] Y. Yuan, J. Fang, and Q. Wang, "Robust superpixel tracking via depth fusion," TCSVT, vol. 24, no. 1, pp. 15-26, 2014

[41] S. Gao, Z. Han, C. Li, Q. Ye, and J. Jiao, "Real-time multipedestrian tracking in traffic scenes via an rgb-d-based layered graph model," IEEE Trans. Intelligent Transportation Systems, vol. 16, pp. 2814-2825, 2015.

[42] L. Zhang, W. Wu, T. Chen, N. Strobel, and D. Comaniciu, "Robust object tracking using semi-supervised appearance dictionary learning," Pattern Recognition Letters, vol. 62, pp. 17-23, 2015.
[43] Z. He, S. Yi, Y. Cheung, X. You, and Y. Y. Tang, "Robust object tracking via key patch sparse representation," IEEE Trans. Cybernetics, vol. 47, pp. 354-364, 2017.

[44] Z. Han, J. Jiao, B. Zhang, Q. Ye, and J. Liu, "Visual object tracking via sample-based adaptive sparse representation (adasr)," Pattern Recognition, vol. 44, pp. 2170-2183, 2011

[45] Z. He, X. Li, X. You, D. Tao, and Y. Y. Tang, "Connected component model for multi-object tracking," TIP, vol. 25, pp. 3698-3711, 2016.

[46] R. Wang, H. Guo, L. S. Davis, and Q. Dai, "Covariance discriminative learning: A natural and efficient approach to image set classification," in $C V P R, 2012$

[47] J. Chien and C. Wu, "Discriminant waveletfaces and nearest feature classifiers for face recognition," PAMI, vol. 24, pp. 1644-1649, 2002.

[48] P. Vincent and Y. Bengio, "K-local hyperplane and convex distance nearest neighbor algorithms," in NIPS, 2001.

[49] Z. Huang, R. Wang, S. Shan, and X. Chen, "Learning euclidean-toriemannian metric for point-to-set classification," in CVPR, 2014.

[50] V. Arsigny, P. Fillard, X. Pennec, and N. Ayache, "Geometric means in a novel vector space structure on symmetric positive-definite matrices," SIAM, vol. 29, pp. 328-347, 2006

[51] M. Danelljan, G. Häger, F. S. Khan, and M. Felsberg, "Accurate scale estimation for robust visual tracking," in $B M V C, 2014$.

[52] H. Possegger, T. Mauthner, and H. Bischof, "In defense of color-based model-free tracking," in CVPR, 2015.

[53] Z. Kalal, K. Mikolajczyk, and J. Matas, "Tracking-learning-detection," TPAMI, pp. 1409-1422, 2012.

[54] X. Jia, H. Lu, and M.-H. Yang, "Visual tracking via adaptive structural local sparse appearance model," in Proceedings of IEEE Conference on Computer Vision and Pattern Recognition, 2012.

[55] T. Zhang, B. Ghanem, S. Liu, and N. Ahuja, "Robust visual tracking via multi-task sparse learning," in Proceedings of IEEE Conference on Computer Vision and Pattern Recognition, 2012.

[56] N. Wang and D.-Y. Yeung, "Learning a deep compact image representation for visual tracking," in Advances in Neural Information Processing Systems, 2013.

[57] T. B. Dinh, N. Vo, and G. G. Medioni, "Context tracker: Exploring supporters and distracters in unconstrained environments," in CVPR, 2011.

[58] A. Adam, E. Rivlin, and I. Shimshoni, "Robust fragments-based tracking using the integral histogram," in CVPR, 2006.

[59] D. A. Ross, J. Lim, R. Lin, and M. Yang, "Incremental learning for robust visual tracking," IJCV, pp. 125-141, 2008.

[60] C. Bao, Y. Wu, H. Ling, and H. Ji, "Real time robust L1 tracker using accelerated proximal gradient approach," in CVPR, 2012

[61] L. Sevilla-Lara and E. G. Learned-Miller, "Distribution fields for tracking," in CVPR, 2012

[62] S. Oron, A. Bar-Hillel, D. Levi, and S. Avidan, "Locally orderless tracking," in CVPR, 2012.

[63] D. Wang, H. Lu, and M. Yang, "Least soft-threshold squares tracking," in $C V P R, 2013$

[64] J. V. Davis, B. Kulis, P. Jain, S. Sra, and I. S. Dhillon, "Informationtheoretic metric learning," in ICML, 2007, pp. 209-216.

[65] E. Mark, G. L. Van, W. C. K. I., W. John, and Z. Andrew, "The pascal visual object classes (voc) challenge," International Journal of Computer Vision, vol. 88, pp. 303-338, 2010.

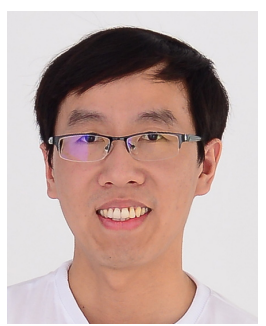

Shengping Zhang received the Ph.D. degree in computer science from the Harbin Institute of Technology, Harbin, China, in 2013. He is currently a Professor with the School of Computer Science and Technology, Harbin Institute of Technology at Weihai. He had been a Post-Doctoral Research Associate with Brown University, Providence, RI, USA, a PostDoctoral Research Associate with Hong Kong Baptist University, Hong Kong, and a Visiting Student Researcher with University of California at Berkeley, Berkeley, CA, USA. He has authored or co-authored over 50 research publications in refereed journals and conferences. His research interests include deep learning and its applications in computer vision. Dr. Zhang is also an Associate Editor of Signal Image and Video Processing. 


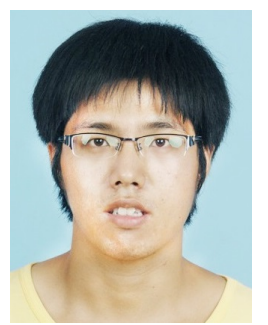

Yuankai Qi received the B.S. and M.S. degrees from Harbin Institute of Technology, China, in 2011 and 2013, respectively, and is currently working toward the Ph.D. degree in computer science and technology at Harbin Institute of Technology, China. His research interests include object tracking, sparse coding, and machine learning.

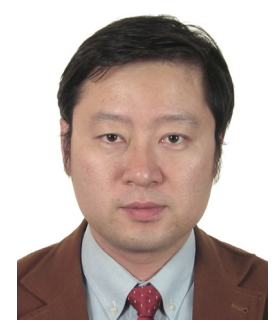

Feng Jiang received the B.S., M.S., and Ph.D. degrees in computer science from Harbin Institute of Technology (HIT), Harbin, China, in 2001, 2003, and 2008, respectively. He is now an Associated Professor in the Department of Computer Science, HIT. He had been a visiting scholar in the School of Electrical Engineering, Princeton University, United States. His research interests include computer vision, image and video processing and pattern recognition.

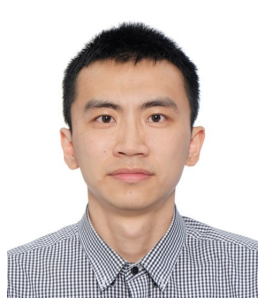

Xiangyuan Lan received the B.Eng. degree in computer science and technology from the South China University of Technology, China in 2012 and the Ph.D. degree in the Department of Computer Science, Hong Kong Baptist University, Hong Kong in 2016. In 2015, he was a Visiting Scholar with the Computer Vision Laboratory, University of Maryland Institute for Advanced Computer Studies, University of Maryland, College Park, MD, USA. $\mathrm{He}$ is now a Post-Doctorial research fellow with Hong Kong Baptist University. Dr. Lan's current research interests include sparse representation and deep learning for computer vision and pattern recognition problems.

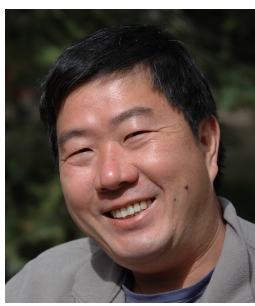

Pong C. Yuen received his B.Sc. degree in Electronic Engineering with First Class Honours in 1989 from City Polytechnic of Hong Kong, and his Ph.D. degree in Electrical and Electronic Engineering in 1993 from The University of Hong Kong. He joined the Hong Kong Baptist University in 1993 and, currently is a Professor and Head of the Department of Computer Science. Dr. Yuen was a recipient of the University Fellowship to visit The University of Sydney in 1996. In 1998, Dr. Yuen spent a 6-month sabbatical leave in The University of Maryland Institute for Advanced Computer Studies (UMIACS), University of Maryland at college park. From June 2005 to January 2006, he was a visiting professor in GRAVIR laboratory (GRAphics, VIsion and Robotics) of INRIA Rhone Alpes, France. Dr. Yuen was the director of Croucher Advanced Study Institute (ASI) on biometric authentication in 2004 and the director of Croucher ASI on Biometric Security and Privacy in 2007. Dr. Yuen has been actively involved in many international conferences as an organizing committee and/or technical program committee member. He was the track co-chair of International Conference on Pattern Recognition (ICPR) 2006 and the program co-chair of IEEE Fifth International Conference on Biometrics: Theory, Applications and Systems (BTAS) 2012. Currently, Dr. Yuen is an Editorial Board Member of Pattern Recognition and Associate Editor of IEEE Transactions on Information Forensics and Security, and SPIE Journal of Electronic Imaging. He is also serving as a Hong Kong Research Grant Council Engineering Panel Member. Dr. Yuen?s current research interests include video surveillance, human face recognition, biometric security and privacy.

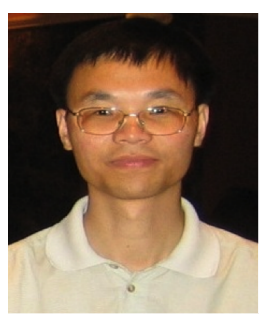

Huiyu Zhou obtained a Bachelor of Engineering degree in Radio Technology from Huazhong University of Science and Technology of China, and a Master of Science degree in Biomedical Engineering from the University of Dundee of United Kingdom, respectively. He was then awarded a Doctor of Philosophy degree in Computer Vision from the HeriotWatt University, Edinburgh, United Kingdom. Dr. Zhou is a lecturer at School of Electronics, Electrical Engineering and Computer Science, Queens University of Belfast, United Kingdom. He has published over 130 peer-reviewed papers in the field. He was the recipient of "Computer Vision and Image Understanding 2012 Most Cited Paper Award", "International Conference on Pattern Recognition Applications and Methods 2016 Best Paper Award" and was nominated for "Medical \& Biological Engineering \& Computing 2006 Nightingale Prize". Four of his papers recently published by Elsevier were ranked as the ScienceDirect Top 25 Articles. Dr. Zhou currently serves as an Associate Editor of "IEEE Transaction on HumanMachine Systems", and is on the Editorial Boards of five refereed journals. 